\title{
Development and Measurement of Fetal Parital Ossification
}

\author{
Weijian Zhang , Jizhong Wang , Wenshuo Wang \\ Department of Bioengineering, Shandong Jianzhu University, jinan,250014, China \\ Department of Mathematics, Shandong Jianzhu University, jinan,250014, China
}

China Police Daily, Ministry of Public Security the People's Republic of China,Peking,100160, China

Keywords: Fetus; Parietal bone; Ossification; Measurement

\begin{abstract}
The paper aims to discover the development rule of fetal parital bone. One hundred and eighty parietal bones of 5-38th weeks old were observed and measured. With the Noback's method every fetus was made transparent and then dyed dark red by the Alizarin red. The measured data are added to differential and integral calculus formula to calculate parietal ossification length and make correlated regression analysis. The results show that the time of ossification of different parts of fetal parietal bones are between the 9th and the 38th week. The parietal sagittal ossification length is $85.12 \pm 1.07 \mathrm{~mm}$ and the coronary ossification length is $82.62 \pm 1.24 \mathrm{~mm}$. from the 37 th to the 38th week, parietal coronary ossification bending appears. from the 13rd to the 14th week with the bending height being $3.74 \pm 0.47$, and sagittal ossification bending occurs from the 17 th to the18th week with the bending height being $1.60 \pm 0.45$. Correlation analysis shows that parietal sagittal ossification length and coronary ossification length have highly positive correlation with gestational age respectively $(r=0.9926,0.9869, \mathrm{P}$ $<0.01$ ). Two regression equations are established, and parietal ossification diameter length can be estimated. It can be concluded that fetal parietal sagittal ossification length is greater than coronary ossification length. Coronary ossification bending appears earlier than sagittal ossification bending but sagittal bending height is greater than coronary bending height.
\end{abstract}

\section{Introduction}

There are some accounts about the study on the primary ossification center and some measurement of the ossified main limb bone of the fetuses [1], but there was no report about the measurement of fetal parietal ossification up to now. We observed and measured the fetal parietal ossification development in order to enrich embryology data.

\section{Materials and Methods}

\section{Preparation of samples}

A total of 180 normally developed fetus samples of 5-38 weeks old were collected. For those fetuses less than 10 weeks old, the fetus age was calculated according to the CR value in the Corliss Table [2] .For the fetuses more than 10 weeks old, the fetus age was calculated according to the CR in Scammon's Table [3]. Transparent samples were prepared and stained with alizarin red bones. The ossified part of the bones was stained red in order to be easily measured. There were 76 males and 74 females in the 11-38 weeks old embryonic specimens, all of whom were donated by the volunteers. 


\section{Measurement method}

Because the samples 5-38 weeks old embryonic transparent specimens, were small and in immersed in glycerin soft and creamy, it is difficult to operate manually with graph paper or tape, therefore we improved our measurement method.

Based on the differential equation, we developed the following formula to calculate the actual length of ossified bones based on the chord length and height, (formula 1), where $l$ represents the actual length of ossified bones, $d$ represents the chord length of the bending bones and $\boldsymbol{h}$ represents the chord height (curved height) [4]. With this formula, we only need to measure the chord length and height of the bending bones to calculate the arc length.

$$
l=\frac{2 h}{a^{2}}\left[a \sqrt{1+a^{2}}+\ln \left(a+\sqrt{1+a^{2}}\right)\right] \quad a=\frac{4 h}{D}
$$

Specific operation method.The chord length $d$ of bending bone ossification part was first measured and than the chord height of the (curved height) $\boldsymbol{h}$ was measured. The chord height $\boldsymbol{h}$ was the straight-line distance from $\boldsymbol{d}$ to the highest point of the curving bones (Fig. 1). The chord length and height were put into formula (1) to calculated the arc length $l$ (Fig.1). Spss 13.0 statistical software was used for data processing.

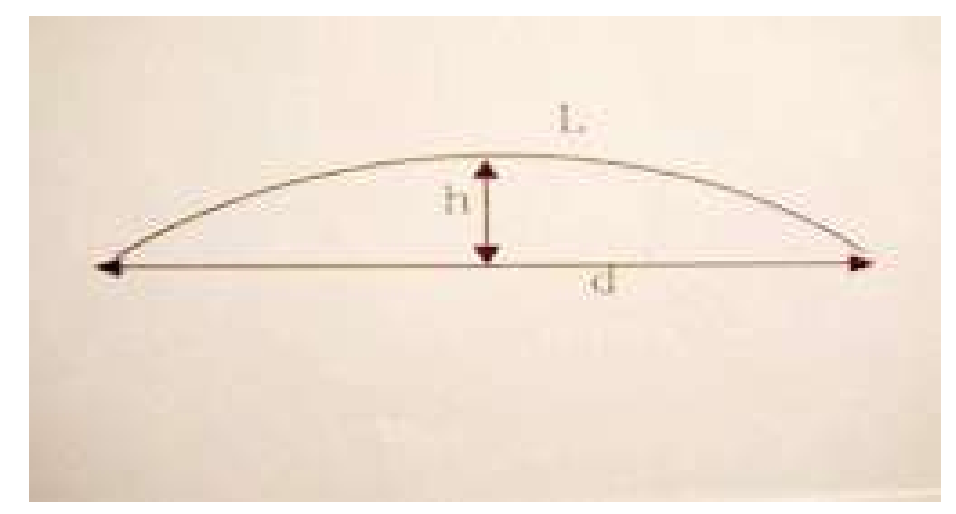

Figure 1 measurement scheme of less than $180^{\circ}$ bent bone

The parietal bone of sagittal and coronary ossification chord length $d$ and curved height $\boldsymbol{h}$ were measured. The data (the chord length and height ) were put into formula(1) to calculated the actual arc length of parietal ossification.

Unified measurements of right parietal were made three times from each measuring point and ave ge was taken. No project comparison and gender comparison were made between left and right lateral parietal bone measurements. The actual parietal sagittal ossification length was the actual ossification arc length from the angulus frontalis to angulus occipitalisc(parietal arc $b-1$ ). The parietal sagittal ossification chord length was the linear distance from the angulus frontalis to ngulus occipitalisc (parietal chord b-1 ). The parietal sagittal ossification curved height $\boldsymbol{h}$ was the vertical distance between chord line and the highest point of the arc measured (parietal subtense) [5]. The parietal coronary calification chord length was measured as the distance from the front upper corner(angulus frontalis) of parietal bone (forehead) to the anterior corner of parietal bone (sphenoidal angle) [6]. The parietal coronary calification arc length was the distance between forehead of parietal bone and the sphenoidal angle. The chord height was the vertical distance between chord line and the highest point of the arc. 
Bone measuring equipment(provided by Beijing Huaxing Ruian Science and Technology Co, Ltd, China, product standards: GB5704.4-85.) was used to measure the chord length and height. Straight Angle gauge(accuracy of $0.05 \mathrm{~mm}$ ) was used to measure chord length (d), and bent three feet parallel ruler (accuracy of $0.05 \mathrm{~mm}$ ) was used to measure curved high (h). SPSS13.0 statistical software was used for all data processing.

\section{Results}

\section{Parietal ossification measurement}

The fetal parietal bone was membranous ossification and there was only one primary ossification center on the parietal tuber. The primary ossification center

appears at the 8th week[7]. The earliest part is parietal tuber and the latest part is inferior temporal line.

i ) Measurement of parietal sagittal ossification length The data of the sagittal chord length $d$ sagittal curved height $\boldsymbol{h}$ and actual length of parietal ossification $l$ are shown in Table 1.

Table 1 Measurement of the fetal parietal sagittal ossification length $(\mathrm{n}=10 \bar{\chi} \pm \mathrm{s} \mathrm{mm})$

\begin{tabular}{|c|c|c|c|c|c|c|}
\hline \multirow{2}{*}{$\begin{array}{l}\text { Fetus age } \\
5 \sim\end{array}$} & \multicolumn{2}{|c|}{$\begin{array}{l}\text { Chord length } d \\
\text { Smallest-largest } \bar{\chi} \pm \mathrm{s}\end{array}$} & \multicolumn{2}{|c|}{$\begin{array}{l}\text { Chord height } \boldsymbol{h} \\
\text { smallest - largest } \bar{\chi} \pm \mathrm{s}\end{array}$} & \multicolumn{2}{|c|}{$\begin{array}{l}\text { Actua llength of bone } \\
\text { ossification } l \\
\text { smallest- largest } \bar{\chi} \pm \mathrm{s}\end{array}$} \\
\hline & 0.00 & 0.00 & 0.00 & 0.00 & 0.00 & 0.00 \\
\hline $7 \sim$ & 0.00 & 0.00 & 0.00 & 0.00 & 0.00 & 0.00 \\
\hline $9 \sim$ & $2.00 \sim 3.50$ & $2.05 \pm 0.85$ & 0.00 & 0.00 & $2.10 \sim 3.50$ & $2.05 \pm 0.85$ \\
\hline $11 \sim$ & $8.50 \sim 9.50$ & $8.97 \pm 0.29$ & 0.00 & 0.00 & $8.50 \sim 9.50$ & $8.97 \pm 0.29$ \\
\hline $13 \sim$ & $18.00 \sim 20.00$ & $18.76 \pm 0.52$ & 0.00 & 0.00 & $18.20 \sim 20.00$ & $18.76 \pm 0.52$ \\
\hline $15 \sim$ & $21.50 \sim 24.00$ & $22.50 \pm 0.63$ & 0.00 & 0.00 & $21.50 \sim 24.00$ & $22.50 \pm 0.63$ \\
\hline $17 \sim$ & $27.50 \sim 30.50$ & $29.11 \pm 1.01$ & $1.00-2.00$ & $1.60 \pm 0.45$ & $27.88 \sim 30.84$ & $29.39 \pm 0.99$ \\
\hline $19 \sim$ & $29.00-34.00$ & $31.91 \pm 0.75$ & $2.20 \sim 3.00$ & $2.70 \pm 0.27$ & $31.90 \sim 34.60$ & $32.51 \pm 0.76$ \\
\hline $21 \sim$ & $39.00-41.00$ & $40.16 \pm 0.47$ & $4.50-6.00$ & $5.61 \pm 0.49$ & $41.19 \sim 42.47$ & $42.15 \pm 0.57$ \\
\hline $23 \sim$ & $45.00-46.50$ & $45.78 \pm 0.37$ & $9.00 \sim 10.00$ & $9.32 \pm 0.39$ & 49.79 51.09 & $50.43 \pm 0.44$ \\
\hline $25 \sim$ & $46.50-49.00$ & $47.20 \pm 0.65$ & $10.00 \sim 12.00$ & $11.20 \pm 0.52$ & $51.72 \sim 54.21$ & $53.75 \pm 1.08$ \\
\hline $27 \sim$ & $50.00 \sim 54.00$ & $51.85 \pm 0.82$ & $12.00 \sim 13.50$ & $12.83 \pm 0.40$ & $57.82 \sim 61.35$ & $59.33 \pm 1.03$ \\
\hline $29 \sim$ & $61.50 \sim 64.00$ & $61.95 \pm 0.87$ & $13.50 \sim 15.00$ & $14.40 \pm 0.39$ & $68.76 \sim 71.91$ & $70.04 \pm 0.88$ \\
\hline $31 \sim$ & $64.00 \sim 67.50$ & $65.96 \pm 0.94$ & $14.00 \sim 16.50$ & $15.54 \pm 0.77$ & $73.35 \sim 76.55$ & $74.64 \pm 0.97$ \\
\hline $33 \sim$ & $68.50 \sim 72.00$ & $69.95 \pm 0.98$ & $14.80 \sim 17.00$ & $15.86 \pm 0.83$ & $77.16 \sim 81.59$ & $78.57 \pm 1.28$ \\
\hline $35 \sim$ & $72.00 \sim 73.80$ & $72.69 \pm 0.45$ & $15.00 \sim 17.10$ & $16.26 \pm 0.78$ & $80.17 \sim 82.95$ & $81.55 \pm 0.76$ \\
\hline $37 \sim$ & $75.00 \sim 77.00$ & $75.53 \pm 0.60$ & $15.50 \sim 18.00$ & $17.36 \pm 0.83$ & $83.28 \sim 87.07$ & $85.12 \pm 1.07$ \\
\hline
\end{tabular}

ii ) Measurement of the fetal coronary calcification length Fetal parietal coronary calcification chord length d, curved height $\boldsymbol{h}$, the actual coronary ossification of arc length $l$ are shown in Table 2. 
Table 2 Measurement of the fetal coronary calcification length $(\mathrm{n}=10 \bar{\chi} \pm \mathrm{s} \mathrm{mm})$

\begin{tabular}{|c|c|c|c|c|c|c|}
\hline \multirow{2}{*}{$\begin{array}{l}\text { Fetus age } \\
5 \sim\end{array}$} & \multicolumn{2}{|c|}{$\begin{array}{l}\text { coronary coronal length } d \\
\text { smallest-largest } \bar{\chi} \pm \mathrm{s}\end{array}$} & \multicolumn{2}{|c|}{$\begin{array}{l}\text { coronary height } h \\
\text { smallest-largest } \bar{\chi} \pm \mathrm{s}\end{array}$} & \multicolumn{2}{|c|}{$\begin{array}{l}\text { Actual coronary length } \\
\text { of bone ossification } l \\
\text { Smallest-largest } \bar{\chi} \pm \mathrm{s}\end{array}$} \\
\hline & 0.00 & 0.00 & 0.00 & 0.00 & 0.00 & 0.00 \\
\hline $7 \sim$ & 0.00 & 0.00 & 0.00 & 0.00 & 0.00 & 0.00 \\
\hline $9 \sim$ & $2.10-3.80$ & $2.99 \pm 0.40$ & 0.00 & 0.00 & $2.10-3.80$ & $2.99 \pm 0.40$ \\
\hline $11 \sim$ & $10.50-13.00$ & $11.16 \pm 0.76$ & 0.00 & 0.00 & $10.50 \sim 13.00$ & $11.22 \pm 0.78$ \\
\hline $13 \sim$ & $18.50-22.00$ & $19.90 \pm 0.96$ & $0.00-6.00$ & $3.74 \pm 0.47$ & $20.05 \sim 23.40$ & $21.47 \pm 1.02$ \\
\hline $15 \sim$ & $22.10-25.00$ & $23.31 \pm 0.70$ & $5.50-7.50$ & $6.49 \pm 0.52$ & $26.46-28.51$ & $27.25 \pm 1.10$ \\
\hline $17 \sim$ & $28.00-30.00$ & $28.76 \pm 0.57$ & $8.30-10.50$ & $9.45 \pm 0.52$ & $35.36 \sim 36.39$ & $35.65 \pm 0.37$ \\
\hline $19 \sim$ & $31.00-34.00$ & $32.74 \pm 0.72$ & $9.50-11.00$ & $10.12 \pm 0.75$ & $37.94-41.50$ & $39.80 \pm 1.11$ \\
\hline $21 \sim$ & $39.50-43.00$ & $41.46 \pm 0.90$ & $9.50-11.50$ & $10.26 \pm 0.71$ & $46.14-48.57$ & $47.46 \pm 0.87$ \\
\hline $23 \sim$ & $47.00-48.50$ & $47.36 \pm 0.52$ & $9.80-12.50$ & $10.78 \pm 0.96$ & $51.98 \sim 56.07$ & $53.38 \pm 1.13$ \\
\hline $25 \sim$ & $48.70-51.00$ & $49.26 \pm 0.79$ & $10.50 \sim 13.00$ & $11.53 \pm 0.51$ & $54.37 \sim 57.55$ & $55.73 \pm 0.98$ \\
\hline $27 \sim$ & $52.00-53.50$ & $53.49 \pm 0.65$ & $10.60 \sim 14.00$ & $12.47 \pm 0.66$ & $58.44-62.52$ & $60.46 \pm 1.09$ \\
\hline $29 \sim$ & $56.90-60.00$ & $58.29 \pm 0.78$ & $13.20-15.00$ & $13.74 \pm 0.57$ & $64.24-68.34$ & $66.11 \pm 1.24$ \\
\hline $31 \sim$ & $63.90-66.00$ & $64.92 \pm 0.49$ & $13.30-15.60$ & $14.25 \pm 0.76$ & $71.29 \sim 73.10$ & $72.25 \pm 0.59$ \\
\hline $33 \sim$ & $66.90 \sim 70.00$ & $68.54 \pm 0.74$ & $13.50-17.00$ & $14.38 \pm 1.07$ & $73.65 \sim 78.49$ & $75.65 \pm 1.37$ \\
\hline $35 \sim$ & $68.00-71.50$ & $69.43 \pm 0.89$ & $14.50-17.50$ & $15.51 \pm 0.48$ & $76.30-79.36$ & $77.80 \pm 0.78$ \\
\hline $37 \sim$ & $73.00-76.00$ & $74.65 \pm 0.85$ & $15.00-18.00$ & $15.66 \pm 0.88$ & $80.53 \sim 84.85$ & $82.62 \pm 1.24$ \\
\hline
\end{tabular}

Regression analysis of correlation between parietal ossification diameter line and gestational age

Correlation analysis shows that the correlation coefficient between gestational age and parietal sagittal ossification length is $0.9869, \mathrm{P}<0.01$ Indicating a highly positive correlation. The correlation coefficient between gestational age and the parietal coronary ossification length is $0.9926, \mathrm{P}<0.01$ showing that fetal parietal bone ossification length has highly positive correlation with gestational age. The regression equation is deduced and shown in Table 3.

\begin{tabular}{|c|c|c|c|}
\hline lines & regression equation & $\mathrm{r}$ & $\mathrm{p}$ \\
\hline $\begin{array}{l}\text { Fetal parietal sagittal } \\
\text { ossification length }\end{array}$ & $y=-20.568+2.9676 x$ & 0.9869 & $<0.01$ \\
\hline $\begin{array}{l}\text { Fetal parietal coronary } \\
\text { ossification length }\end{array}$ & $y=-15.709+2.7924 x$ & 0.9926 & $<0.01$ \\
\hline
\end{tabular}

\section{Discussion}

\section{Parietal bone development rule}

The results show that the forming time of fetal parietal primary ossification center of Chinese is basically the same as that of American[8]. The various parts of the parietal bone osseous marks appear from the 9th to the 38th week and the two parietal bones are still not fully healed when the baby is born[7]. 
Table 2-1 and Table 2-2 show parietal ossification begins since the 9th week of embryonic development.From the $13 \mathrm{rd}$ to the 14 th week, coronary ossification bending appears, and between the 37 th and the 38 th week, coronary average height is $15.66 \pm 0.88 \mathrm{~mm}$. From the 17 th to the 18 th week, parietal sagittal ossification bending appears.and between the 37th and the 38th week, coronary average bending height is $17.36 \pm 0.83 \mathrm{~mm}$.Parietal coronary bending appear is earlier than sagittal bending but the sagittal bending is higher then coronary bending. From the 37 th to the 38th week, average parietal sagittal chord length is $75.53 \mathrm{~mm}$, and the actual sagittal ossification arc length is $85.12 \mathrm{~mm}$. Average coronary ossification chord length is $74.65 \pm 0.85 \mathrm{~mm}$, and the actual coronary ossification length is $82.62 \mathrm{~mm}$. From the 9th to the 25th week, the parietal sagittal ossification length is less than the coronary calcification length, and in the 27 th week, both are basically equal. From the 29th to the 37th week, the parietal sagittal ossification length is greater than the coronary calcification length with an average of $85.12 \mathrm{~mm}$.

\section{Analysis of correlation between parietal ossification development and gestational age}

Table 3 shows parietal sagittal ossification length and coronary ossification length have highly positive correlation with gestational age respectively. The two deduced regression equations can be used to roughly estimate the parietal bone ossification diameter line length according to gestational age.

\section{Use of new measurment method}

Acoording to traditional measurement method, adult skull specimens needed to be first put in the cranial fixed device. The skull of the primeter, arc and chord measurement point were determined acoording to traditional method[9], and graph paper and tape were used to make manual measurement, which needs people to cooperate to complete. Traditional graph paper or tape are often used to measure curved bone arc length manually [10]. This method is suitable for dry adult skull specimen measurement. The transparent specimens in the study are immersed in glycerin, soft and creamy, and it is difficult to ensure the accuracy of the manual measurement by using graph paper or tape, so the new measurement method is adopted, which is simple, can save manpower and improve significantly measurement accuracy and speed.

\section{Conclusions}

One hundred and eighty parietal bones of 5-38th weeks old were observed and measured. The time of ossification of different parts of fetal parietal bones are between the 9th and the 38th week. The parietal sagittal ossification length is $85.12 \pm 1.07 \mathrm{~mm}$ and the coronary ossification length is $82.62 \pm 1.24 \mathrm{~mm}$. Correlation analysis shows that parietal sagittal ossification length and coronary ossification length have highly positive correlation with gestational age respectively $(r=0.9926,0.9869, P$ $<0.01)$. the new measurement method is adopted, which is simple, can save manpower and improve significantly measurement accuracy and speed.

\section{Acknowledgments}

This work was undertaken with funds of Fetal Bone System Development Research of Ministry of Health of Shandong Province (No 2001CAICGAI). So, I want to extend my sincere thanks to them. 


\section{References}

[1] Fu Mao, Xiu-fen wu, Sun Zh. The whole weeks of fetal main limbs which to grow and develop[J]. Sichuan Journal of Anatomy1999, 7(26): 223 225.

[2] Corliss C E. 1976. Patten's Human Embryology of Clinical development. New York: Mc Graw-Hill. p 10-11.

[3] Scammon R E. 1973. Two simple nomographs for estimating the age and some of the major external dimensions of the human fetus. Anat Rec 68(2):221-224.

[4] Wang Ji-zhong, Zhang Wei-jian. The measurement method of fetal irregularly curved bones[P]. The Patent of China,200610070187. 8,2007-5-9.

[5] Shao Xiang-qing.1984.Handbook for the measurement of human body. Shanghai: Cishu Press. $p$ 84-85.

[6] Zhang Yan. 1964. Human anatomy. Beijing People's Health Press. p 29-30.

[7] Zhang Wei-jian. Fetal brain skull primary ossification center of observation[J]. Linyi medical college journals:1996, 18 (2) : 95 97.

[8] Potter E. Pathology in fetus and infants(Translated by the pathological anatomy of the group oBeijing second medical collegef) [M]. People's medical publishing house 1982: 12 15.

[9] Wu Ru-kang. 1984. Measurement of human body. Beijing: Scientific Press. p75-95.

[10] Song Wen. 1998. Measurement of arc of chord for adult skulls. Journal of Zhangjiakou Medical College 16: 32. 